\title{
Re: Role of Telomeres and Telomerase in Cancer
}

\author{
Shay JW, Wright WE \\ University of Texas Southwestern Medical Center, Department of Cell Biology, Dallas, USA \\ Semin Cancer Biol. 2011;21:349-353. doi: 10.1016/j.semcancer.2011.10.001. Epub 2011 Oct 17.
}

\section{EDITORIAL COMMENT}

The most important difference between cancer and normall cells is the ability to continuous proliferation. This activation works due to telomeres and telomerase enzyme. Fifty years ago, Leonard Hayflick discovered that cultured normal humans cells have a limited capacity to divide. Today, this withdrawal from the cell cycle after a certain number of cellular divisions (replicative senescence) is known to be triggered as a result of shortened telomeres. Studies on telomeres and telomerase have begun to provide additional information about aging and cancer development and have created new opportunities in the field of regenerative medicine for telomeropathies. Progressive telomere shortening from cell division (replicative aging) provides a barrier for tumor progression. Continuous cell growth in malignancy correlates with the reactivation of telomerase. Telomerase is a cellular reverse transcriptase that adds new deoxyribonucleic acid (DNA) onto the telomeres that are located at the ends of chromosomes. Telomeres consist of many kilobases of TAGGG nucleotide repeats. The telomeric nucleotide repeats shorten with each cell division due to replication problems (DNA repair) and oxidative damage. Quiescent/senescent state of the cell bypass can be accomplished by abrogating cell cycle checkpoint genes (such as TP53, p16INK4a, pRb). Telomerase is detected in approximately 90\% of all malignant tumors. This telomerase activation has emerged as a target for cancer treatment. Telomerase therapeutics are classified as gene therapy (hTERT-telomerase catalytic protein component, hTR-telomerase functional), immunotherapy (Imetalstat-telomerase template antagonist), and small molecule inhibitors. In the near future, more specific researches on telomers and telomerase will contribute to aging/immortality studies (as stem cells) and to discover new biomarkers for malignant tissue or anticancer therapeutics.

Fehmi Narter, MD

\section{Re: Cell Adhesion Molecules and Their Relation to (Cancer) Cell Stemness}

\author{
Farahani E, Patra HK, Jangamreddy JR, Rashedi I, Kawalec M, Rao Pariti RK, Batakis P, Wiechec E \\ Linköping University, Cell Biology Building, Department of Clinical and Experimental Medicine, Division of Cell Biology and Integrative Regenerative Medicine \\ Center, Linköping, Sweden
}

Carcinogenesis. 2014;35:747-759. doi: 10.1093/carcin/bgu045. Epub 2014 Feb 15.

\section{EDITORIAL COMMENT}

How can tumor cells adhere to the surrounding tissues and form a progression or metastasis? Nowadays, there are many researches in the literature about this topic including adhesion molecules cell-adhesion molecules (CAMs). Cancer development and progression are aided by the environmental or genetic/epigenetic factors. As we know, not all cancer cells within tumors have equal tumor growth (the concept of cancer stem cells-CSCs and tumor cell heterogeneity) and it is a key factor for developing new anticancer therapeutics. CAMs have a major role in the interaction between CSC and extracellular matrix (ECM). Cadherins, integrins, selectins and members of immunoglobulin family constitute the major groups of CAMs. CAMs play an important role in cell-to-cell and cell-to-ECM anchoring by maintaining cell and tissue structure, cell signaling and tissue repair. They are dominant in the epithelial-to-mesenchymal transition, cell migration, gene regulation (as $\beta$-catenin/Wnt), cell proliferation, differentiation, wound healing, immune response, and regulation of inflammation. These molecules and transcription factors (twist, snail) have effects on progression and metastasis. Neoangiogenesis is equally responsible in the progression and metastasis. Recently, researches are focused on discovering new antiangiogenic or antiadhesive therapeutics, such as bevacizumab, avastin, integrin inhibitors, and cadherin inhibitors. In the near future, more specific researches on adhesion molecules will provide opportunity for discovering new anticancer therapeutics.

Fehmi Narter, MD 